\title{
Investigation of the Transverse Spread of Erbium Ions Implanted in SOI
}

\author{
Xifeng Qin ${ }^{1}$, Panpan Jiao ${ }^{1}$, Xiaodi Lu$^{2}$, Jinhua Zhao ${ }^{1}$ and Qiao Zhuang ${ }^{1, *}$ \\ ${ }^{1}$ College of Science, Shandong Jianzhu University, Jinan Shandong, China \\ ${ }^{2}$ Shandong Institute for Endemic Disease Control and Prevention, Jinan Shandong, China \\ ${ }^{*}$ Corresponding author
}

\begin{abstract}
The Er (erbium) ions at energies of 200 - $500 \mathrm{keV}$ and dose of $2 \times 10^{15}$ ions $/ \mathrm{cm}^{2}$ were implanted into the optical waveguide material SOI (Silicon - on - insulator) at room temperature ( RT ) under the angles of $0^{\circ}, 45^{\circ}$ and $60^{\circ}$, respectively. The range distribution of $200-500 \mathrm{keV}, 2 \times 10^{15}$ ions $/ \mathrm{cm}^{2}$ Er ions implanted in SOI samples were measured by Rutherford backscattering (RBS) technique. The transverse distribution of 200 - $500 \mathrm{keV} \mathrm{Er}$ ions in SOI samples were calculated according to the experimental principle proposed by Seijiro Furukawa et al. The measured results are compared with Monte Carlo code, SRIM (Stopping and Range of Ions in Matter) predictions. It is found that the measured experimental values are in good agreement with the SRIM 2012 theoretical simulation results.
\end{abstract}

Keywords-electro-optical materials; transverse distribution; Rutherford backscattering technique; ion implantation

\section{INTRODUCTION}

Ion implantation is one of the important production process of semiconductor integrated circuit, optoelectronic devices and integrated optics. It has been widely used in integrated optics, magnetic materials, surface physics, chemistry, medicine, metallurgy and other fields, as well as the manufacturing process of various devices. Er's characteristic emission at 1.54 $\mu \mathrm{m}$, a standard telecommunication wave length, has important significance for optical interconnection and optoelectronic integrated application of optical fiber communication. Photoluminescence (PL) and range profiles of Er doped semiconductors has widely been studied [1-9], ever since Ennen and Schneider [1] first reported on the observation of $1.54 \mu \mathrm{m}$ PL due to $\mathrm{Er}$ in GaAs and $\mathrm{Si}$. With the continuous development of integrated circuit, integrated optics and optoelectronic devices, the traditional $\mathrm{Si}$ materials showed some limitations in these areas because of its performance defects. SOI material has good resistance to high temperature, low power consumption, high speed, low voltage and other advantages. It is a key technology that can break through the limitation of bulk silicon material and silicon integrated circuit, and solve the power crisis of VLSI. SOI:Er is one of the prospective materials for light sources emitting at $1.54 \mu \mathrm{m}$ at RT. In recent years, some works have been done on the projected range distribution, annealing behavior and photoluminescence of Er ions implanted into SOI material[1011]. But the transverse distribution of Er ions implanted into SOI has not attracted sufficient attention. As far as we know, there has been no report on the research of the lateral spreads of $\mathrm{keV}$ Er ions implanted in SOI.
In this paper, the $200-500 \mathrm{keV}, 2 \times 10^{15}$ ions $/ \mathrm{cm}^{2}$ Er ions were implanted into SOI samples with different angles. The depth distributions and the transverse spreads of implanted Er ions at the energy of $200-500 \mathrm{keV}$ in SOI were obtained by RBS technique. The measured transverse spreads are compared with those obtained by SRIM2012 (Stopping and Range of Ions in Matter, the predictions from Monte Carlo code) [12].

\section{EXPERIMENT}

The SOI Smart Cut ${ }^{\circledR}$ UNI-BOND wafers used in the work consisted of a $300 \mathrm{~nm}$ thick crystalline Si cap layer, on a 500 nm thick buried $\mathrm{SiO}_{2}$ layer, and a $720 \mu \mathrm{m}$ thick Si substrate. The implantation is performed on the $500 \mathrm{keV}$ ion implanter at the Institute of Semiconductors, Chinese Academy of Sciences. The SOI samples were cleaned by the standard procedure before implantation. Er ions with energies of $200-500 \mathrm{keV}$ at the dose of $2 \times 10^{15}$ ions $/ \mathrm{cm}^{2}$ were implanted into the SOI samples at RT at the angles of $0^{\circ}, 45^{\circ}$ and $60^{\circ}$. The voltage (it decided the energy of the implanted ions) of the implanter was calibrated by high-tension voltmeter. To avoid excessive heating of the samples, the incident current density was less than $1 \mu \mathrm{A} / \mathrm{cm}^{2}$.

The RBS experiments were performed at 1.7 MV tandem accelerator of the Shandong University. The depth range of Er ions implanted in SOI was measured by the RBS technique. The RBS spectrometry was carried out using $2.1 \mathrm{MeV} \mathrm{He}^{2+}$ ion beam produced by a $1.7 \mathrm{MV}$ tandem accelerator with a ion beam current about $10 \mathrm{nA}$. The SOI samples were fixed on a three axis goniometer actuated by pulse motor in a vacuum chamber. All the measurements were carried out in a vacuum of $2.66 \times 10^{-4} \mathrm{~Pa}$. The backscattering spectra were performed by a multi-channel analyzer.

\section{RESUlTS AND DISCUSSION}

The projection range distribution of Er ions with low energy implanted in SOI was nearly Gaussian, so the deepness profile could be depicted by the projected range and the range straggling, the standard deviation of the Gaussian distribution in deepness. The range straggling has been calculated from the surveyed full width at half maximum (FWHM)[13].

According to the composition by Furukawa and Matsumura[14], the transverse spread of an deposited ion in SOI can be put into the depth range straggling of a target under canted implantation. If the spatiality probability distribution of the implanted ions is a three-dimensional Gaussian, the deepness distribution for a target tilted at an angle to the 
incident ion beam also becomes a Gaussian with standard straggling $\Delta D$. The transverse spread and range straggling (longitudinal straggling) were denoted by $\Delta X_{\mathrm{L}}$ and $\Delta R_{\mathrm{p}}$, respectively. Then the transverse distribution could be obtained by using Furukawa's formula[14]:

$$
(\Delta D)^{2}=\left(\Delta R_{\mathrm{p}}\right)^{2} \cos ^{2} \theta+\left(\Delta X_{\mathrm{L}}\right)^{2} \sin ^{2} \theta
$$

The transverse spread $\Delta X_{\mathrm{L}}$ can be calculated from two measurements of $\Delta D$ for two different tilted implantation angles $\theta$. Generally speaking, the deepness distributions of the implanted ions are not Gaussian absolutely; only in a small incident energy range of the implanted ion is distributed close to a Gaussian. Hence, Furukawa and Matsumura's method can only be approximately correct.

Figure 1 shows the RBS spectrum of $400 \mathrm{keV}, 2 \times 10^{15}$ ions $/ \mathrm{cm}^{2}$ Er ions implanted in SOI sample at an angle of $45^{\circ}$ at RT. The energy of the $\mathrm{He}$ ion is $2.1 \mathrm{MeV}$, and the backscattering angle is $165^{\circ}$.

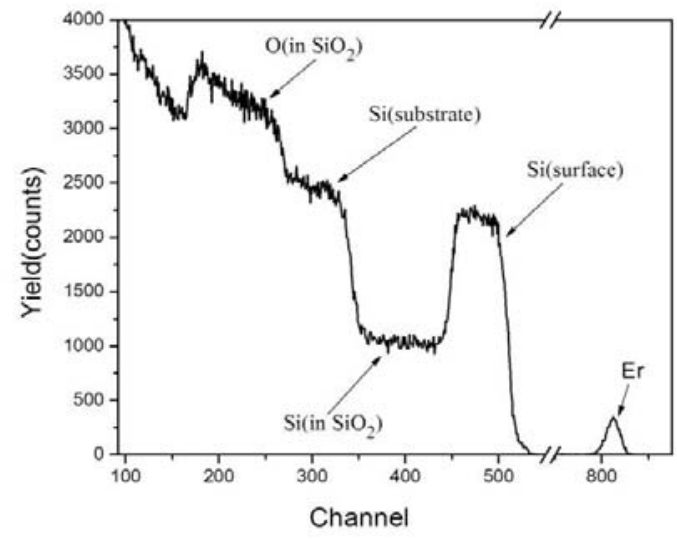

FIGURE I. RBS SPECTRUM OF $400 \mathrm{keV}, 2 \times 10^{15} \mathrm{ions} / \mathrm{cm}^{2}$ Er IONS IMPLANTED IN SOI SAMPLE AT $45^{\circ}$

For the convenience of measurement, calculation, the depth distributions of RBS spectra of $400 \mathrm{keV}, 2 \times 10^{15}$ ions $/ \mathrm{cm}^{2} \mathrm{Er}$ ions implanted in SOI samples at $0^{\circ}$ and $45^{\circ}$ are presented in Figure 2. The distribution shapes denoted by circles and triangles correspond to the samples were performed at $0^{\circ}$ and $45^{\circ}$ incident angles of the implanted ion beam, respectively. The Gaussian fit curve is represented by the solid line. It is evident that both distribution shapes show nearly Gaussian profiles for the incident ions. The values of the range straggling $\Delta R_{\mathrm{p}}\left(0^{\circ}\right)$ and $\Delta D_{1}\left(45^{\circ}\right)$ can be derived from the measured FWHM after taking into account the energy resolution of the measuring system and the energy straggling of He ions in the target. It is seen that the peak position of the distribution of implanted ions shifts toward the surface with an increasing angle $\theta$. The profile shapes closely resemble each other except for a difference in FWHM. The FWHM becomes narrower with increasing the tilted angle. The main step in data analysis is the conversion of the RBS energy spectra to depth profiles. This work was done by using the theory of Chu, Mayer, and
Nicolet [13]. The values of $\Delta R_{\mathrm{p}}$ and $\Delta D_{1}$ are $39.8 \mathrm{~nm}, 33.1$ $\mathrm{nm}$, respectively. Using Furukawa's formula (1) we get the lateral spread $\Delta X_{\mathrm{L}}$ of $400 \mathrm{keV}$ Er ions implanted in SOI sample, $\Delta X_{\mathrm{L} 1}=24.5 \mathrm{~nm}$.

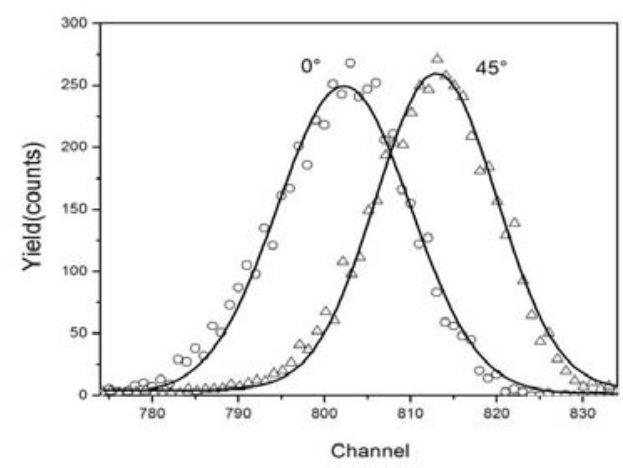

FIGURE II. DEPTH DISTRIBUTIONS OF RBS SPECTRA OF $400 \mathrm{keV}$, $2 \times 1015 \mathrm{ions} / \mathrm{cm} 2$ Er IONS IMPLANTED IN SOI SAMPLE AT $0^{\circ}$ AND $45^{\circ} \mathrm{AND}$ SOLID LINE REPRESENTS THE GAUSSIAN FIT

Figure 3 represents the RBS spectrum of $400 \mathrm{keV}, 2 \times 10^{15}$ ions $/ \mathrm{cm}^{2}$ Er ions implanted in SOI sample at $60^{\circ}$. The energy of the $\mathrm{He}$ ion is $2.1 \mathrm{MeV}$, and the backscattering angle is $165^{\circ}$.

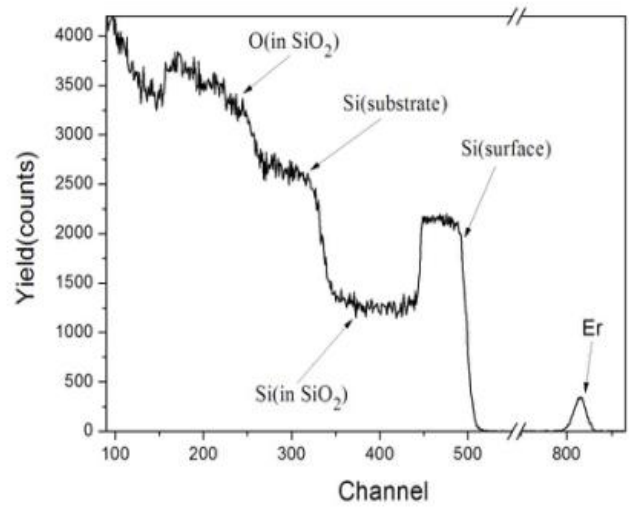

FIGURE III. RBS SPECTRUM OF $400 \mathrm{keV}, 2 \times 1015$ ions/cm2 Er IONS IMPLANTED IN SOI SAMPLE AT $60^{\circ}$

Figure 4 shows the depth distributions of RBS spectra of $400 \mathrm{keV}, 2 \times 10^{15}$ ions $/ \mathrm{cm}^{2}$ Er ions implanted in SOI sample at $0^{\circ}$ ( circles ) and $60^{\circ}$ ( triangles ). It also gives the Gaussian fit curves (solid lines). It can be seen that the distribution shape shows nearly Gaussian profiles for the $60^{\circ}$ implanted Er ions. Same, using the surface energy approximation [13], the range straggling $\Delta D$ of the $60^{\circ}$ implanted Er ions is calculated, $\Delta D_{2}$ $=33.4 \mathrm{~nm}$. Then another value of the transverse spread $\Delta X_{\mathrm{L}}$ of $400 \mathrm{keV}$ Er ions implanted in SOI sample was calculated by using formula (1), $\Delta X_{\mathrm{L} 2}=25.3 \mathrm{~nm}$. 


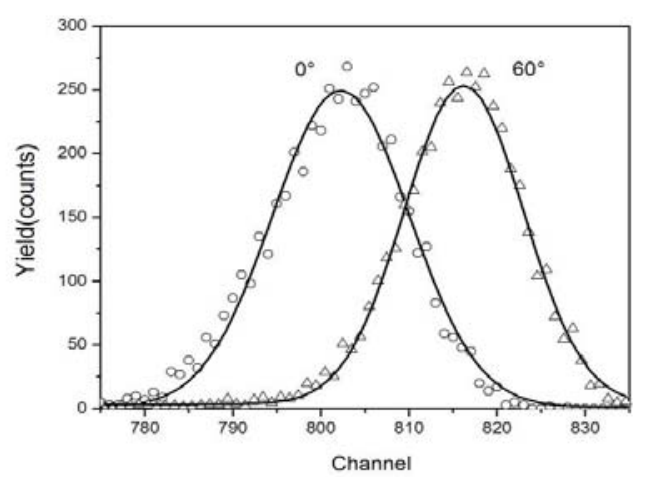

FIGURE IV. DEPTH DISTRIBUTIONS OF RBS SPECTRA OF $400 \mathrm{keV}$, $2 \times 10^{15} \mathrm{ions} / \mathrm{cm}^{2}$ Er IONS IMPLANTED IN SOI SAMPLE AT $0^{\circ}$ AND $60^{\circ}$ AND SOLID LINE REPRESENTS THE GAUSSIAN FIT

The mean experimental value of the transverse spread $\Delta X_{\mathrm{L}}$ of $400 \mathrm{keV}, 2 \times 10^{15}$ ions $/ \mathrm{cm}^{2}$ Er ions implanted in SOI samples is calculated from the data of $\Delta X_{\mathrm{L} 1}$ and $\Delta X_{\mathrm{L} 2}, \Delta X_{\mathrm{L}}$ $=24.9 \mathrm{~nm}$.

One of the primary aim of this work is to compare the experimental transverse spread with its theoretic value. The lateral spread $\Delta X_{\mathrm{L}}$ have been calculated by using SRIM2012 code. The value of the lateral straggling of $400 \mathrm{keV}$ Er ions implanted in SOI calculated by SRIM2012 is $23.6 \mathrm{~nm}$. Compare the theoretical simulation value with the experimental data, it can be seen that the experimental value of the transverse spread of $400 \mathrm{keV}$ Er ions implanted in SOI is a bit of larger than the theoretical simulation data. The difference between the theoretical value of SRIM2012 and the experimental result is about $5.2 \%$, which is a good agreement.

A summary of our experimentally determined values of $\Delta X_{\mathrm{LE}}$ for all implant energies $(200 \mathrm{keV}, 300 \mathrm{keV}, 400 \mathrm{keV}$ and $500 \mathrm{keV}$ ) is shown in Table 1 , together with values $\Delta X_{\mathrm{LS}}$ obtained from SRIM2012. As seen in Table 1, the maximum difference between the experimental results and that of SRIM2010 is $7.8 \%$.

TABLE I. SUMMARY OF THE MEASURED VALUES $\Delta X_{\text {LE }}$ AND THE CALCULATED VALUES $\Delta X_{\mathrm{LS}}$ OF $\Delta X_{\mathrm{L}}$

\begin{tabular}{|c|c|c|c|}
\hline$E / \mathrm{keV}$ & $\Delta X_{\mathrm{LE}} / \mathrm{nm}$ & $\Delta X_{\mathrm{LS}} / \mathrm{nm}$ & $D$ \\
\hline 200 & 15.3 & 14.1 & $7.8 \%$ \\
\hline 300 & 20.2 & 18.9 & $6.4 \%$ \\
\hline 400 & 24.9 & 23.6 & $5.2 \%$ \\
\hline 500 & 28.3 & 26.7 & $5.7 \%$ \\
\hline
\end{tabular}

IV. SUMMARY

200-500 keV, $2 \times 10^{15}$ ions $/ \mathrm{cm}^{2}$ Er ions were implanted in different SOI samples at room temperature at the angles of $0^{\circ}$, $45^{\circ}$ and $60^{\circ}$, respectively. The distribution and the transverse distribution of Er ions implanted in SOI were measured by RBS technique. All deepness distributions of the $200-500 \mathrm{keV}$
Er ions implanted in SOI samples at $0^{\circ}, 30^{\circ}$ and $45^{\circ}$ are approximately Gaussian. The transverse spreads $\Delta X_{\mathrm{L}}$ of 200 $500 \mathrm{keV}$ Er ions implanted in SOI sample were calculated using Furukawa's formula (1) and the data of Chu, Mayer and Nicolet. The experimental values of the transverse spread of the 200-500 keV Er ions implanted in SOI sample are compared with its data of SRIM2012 prediction. It is shown that the experimental transverse spreads agree well with the theoretical values.

\section{ACKNOWLEDGMENT}

This research was financially supported by the National Natural Science Foundation of China (Grant No. 11374193), the Natural Science Foundation of Shandong Province, China (Grant No. ZR2015AM021, ZR2017MA052), the Shandong Jianzhu University Doctoral Foundation, China (Grant No. XNBS1341).

\section{REFERENCES}

[1] H. Ennen, J. Schneider, G. Pomrenke, A. Axmann, " $1.54 \mu \mathrm{m}$ luminescence of erbium implanted III - V semiconductors and silicon," Appl. Phys. Lett. vol. 43, pp. 943-947, August 1983.

[2] R. Lo Savio, M. Miritello, P. Cardile et al., "Concentration dependence of the Er3+ visible and infrared luminescence in Y2-xErxO3 thin films on Si,” J Appl Phys. vol. 106, pp. 043512-1-10, April 2009.

[3] M. Makarova, V. Sih, Joe Warga, et al., "Enhanced light emission in photonic crystal nanocavities with erbium-doped silicon nanocrystals," Appl Phys Lett. vol. 92, pp. 161107-1-3, April 2008.

[4] Jiuyang He, Yuanyuan Ma, Ying Wan et al., "1540 nm photoluminescence enhancement in Er doped $\beta-\mathrm{FeSi} 2 / \mathrm{Si}$," Laser \& Optoelectronics Progress, vol. 52, pp. 83101-1-4, May 2015.

[5] A. Polman, "Erbium implanted thin film photonic materrials," Appl. Phys. vol. 82, pp. 1 - 39, March 1997.

[6] X.F. Qin, M. Chen, X.L. Wang, et al., "Study on preventing segregation of erbium atoms to Si surface by annealing in oxygen atmosphere at high temperature," Nucl. Instrum. Meth B. vol. 268, pp. 1585-1587, May 2010.

[7] M.F. Cerqueira, M. Losurdo, T. Monteiro et al., "Visible and infrared photoluminescence from erbium-doped silicon nanocrystals produced by rf sputtering,” Phys. Stat. Sol. A. vol. 204, pp. 1769-1774, October 2007.

[8] P.G. Kik, A. Polman, "Exciton-erbium energy transfer in Si nanocrystal -doped SiO2," Materials Science and Engineering B. vol. 81, pp. 3-8, may 2001.

[9] X.F. Qin, Y.J. Ji, F.X. Wang, et al., "Measurement of the range distribution and straggling of Er ions implanted in Si crystal," Journal of Shandong Jianzhu University, vol. 24, pp. 212-214, September 2009.

[10] M. Galli, A. Politi, M. Belottiet al., "Strong enhancement of Er3+ emission of room temperature in silicon-on-insulator photonic crystal waveguides,” Appl. Phys. Lett. vol. 81, pp. 241114-1-3, October 2006.

[11] X.F. Qin, G.J. Ma, S.H. Shi, et al., "Investigation of range distribution of Er ions implanted in SOI," Acta Phys. Sin. vol. 63, pp. 176101-1-5, May 2014.

[12] Information on http://www.srim.org.

[13] W.K. Chu, J.W. Mayer, M.A. Nicolet, Backscattering Spectrometry, Academic press, New York, 1978, pp. 134-156.

[14] S. Furukawa and H. Matsumyra, "Backscattering study on lateral spread of implanted ions," Appl. Phys. vol. 22, pp. 97-98, March 1973. 